\title{
Clinical features and prognosis of COVID-19 in people with spinal cord injury: a case-control study
}

\author{
S. D'Andrea $\circledast^{1,2} \cdot$ O. Berardicurti ${ }^{3} \cdot$ A. Berardicurti ${ }^{1} \cdot$ G. Felzani ${ }^{2} \cdot$ F. Francavilla ${ }^{1} \cdot$ S. Francavilla ${ }^{1} \cdot$ R. Giacomelli ${ }^{3}$. \\ A. Barbonetti ${ }^{1}$
}

Received: 5 June 2020 / Revised: 23 July 2020 / Accepted: 26 July 2020

(c) The Author(s), under exclusive licence to International Spinal Cord Society 2020

\begin{abstract}
Study design Observational case-control study.

Objective Individuals with spinal cord injury (SCI) develop systemic physiological changes that could increase the risk of severe evolution of coronavirus disease 2019 (COVID-19) and result in atypical clinical features of COVID-19 with possible delay in both diagnosis and treatment. We evaluated differences in clinical features and evolution of COVID-19 between people with SCI and able-bodied individuals.

Setting The study was conducted in an Italian inpatient rehabilitation referral center for individuals with SCI during the lockdown for the COVID-19 pandemic.

Methods We compared clinical information between patients with SCI and able-bodied healthcare workers of the same center who tested positive for severe acute respiratory syndrome coronavirus 2 (SARS-CoV-2) in the nasopharyngeal swab polymerase chain reaction.

Results Overall, 15 out of the 25 SCI patients admitted to the center and 17 out of the 69 healthcare workers tested positive for SARS-CoV-2. Patients with SCI exhibited a significantly more advanced age and a higher prevalence of comorbidities. Nevertheless, no significant differences in clinical expression of COVID-19 and treatment strategies were observed between the two groups. All hospitalized subjects were treated in nonintensive care units and no deaths occurred in either group.

Conclusions This study does not support the supposed notion that COVID-19 could exhibit atypical clinical features or a worse evolution in the frail population of people with SCI.
\end{abstract}

\section{Introduction}

Severe acute respiratory syndrome coronavirus 2 (SARSCoV-2) is a new zoonotic viral pathogen that was detected for the first time in December 2019 in China [1]. This agent induces a respiratory tract disease known as coronavirus disease 2019 (COVID-19) that, in some cases, requires a

These authors contributed equally: S. D’Andrea, O. Berardicurti

S. D'Andrea

dandrea.settimio@alice.it

1 Andrology Unit, Department of Life, Health and Environment Sciences, University of L'Aquila, L'Aquila, Italy

2 Spinal Unit, San Raffaele Institute of Sulmona, Sulmona, Italy

3 Rheumatology Unit, Department of Biotechnological and Clinical Applied Sciences, University of L'Aquila, L'Aquila, Italy highly specialized management in intensive care unit [2]. The latest epidemiological data from the European Centre for Disease Prevention and Control indicate an overall mortality rate for COVID-19 of 4.8\% worldwide [3] but the prognosis is worse in people with comorbidities, including type 2 diabetes mellitus, cardiovascular diseases, hypertension, and chronic lung diseases [2]. Male gender and older age have been suggested as possible additional risk factors [2].

It has been hypothesized that people with spinal cord injury (SCI) would be at increased risk of morbidity and mortality by COVID-19 [4, 5]. Dramatic changes in respiratory physiology are registered following SCI. The SCI-related loss of innervations to the abdominal and intercostal muscles leads to noneffective cough and the respiratory muscle weakness results in reduced lung volumes and flow rates $[4,5]$. Indeed, chronic respiratory infections represent one of the most common causes of death in SCI [6]. Furthermore, an increased risk to develop cardiovascular [7] and metabolic diseases [8-10] has been 
well documented in people with SCI, thus making even more plausible a poorer COVID-19 prognosis in this population. Nevertheless, according to the scanty available data from an Italian case report [11] and a Spanish series of seven SCI patients [12], COVID-19 evolution seems to be not so dramatic as expected.

On March 11, 2020, the World Health Organization declared the COVID-19 pandemic [13]. After the Italian lockdown declaration, although all patients with SCI admitted to our inpatient rehabilitation center were promptly isolated and specific protective equipment against the infection was used by the healthcare workers and patients, it was inevitable that cases of COVID-19 would occur. Therefore, in this historic cohort study, we selected all individuals who tested positive for SARS-CoV-2 among people with SCI and able-bodied healthcare workers within the same center, in order to evaluate whether or not clinical course of COVID-19 can be more severe in people with SCI than in able-bodied individuals.

\section{Materials and methods}

\section{Study population and design}

All individuals with SCI admitted to the inpatient rehabilitation center "San Raffaele" of Sulmona (Italy), before the national lockdown declaration (March 9, 2020), as well as healthcare workers of the same center, independently of clinical manifestations, and underwent nasopharyngeal swab polymerase chain reaction testing for SARS-CoV-2. Therefore, all SARS-CoV-2-positive individuals underwent a close medical follow-up and early treatment. All individuals who tested positive for SARS-CoV-2 among people with SCI (cases) and able-bodied healthcare workers (controls) were enrolled in a historic cohort study, carried out according to the strengthening the reporting of observational studies in epidemiology statement [14].

\section{Clinical examination}

On admission to the rehabilitation center, patients with SCI had been subjected to detailed clinical and neurological examination according to the International Standards for Neurological Classification of SCI from the American Spinal Injury Association and Impairment Scale [15]. The degree of functional independence was assessed by the Spinal Cord Independence Measure, version III [16]. During the clinical examination, patients were made to wear light clothing and weighed using a professional mechanical chair scale, model DM2 (Wunder SA BI Srl, Monza, Italia). Patients' height was determined using an elastic tape, measuring the lengths between heel to knee, knee to hip, and hip to head. Body mass index (BMI) $\left(\mathrm{kg} / \mathrm{m}^{2}\right)$ was also calculated. According to previously reported SCIappropriate criteria [8], metabolic syndrome was defined as BMI $\geq 22$ and two or more of the following features: triglycerides $\geq 150 \mathrm{mg} / \mathrm{dL}$ (or current treatment), $\mathrm{HDL}<$ $50 \mathrm{mg} / \mathrm{dL}$, hypertension (or current treatment), fasting glucose $\geq 100 \mathrm{mg} / \mathrm{dL}$, or diabetes mellitus type 2 . The presence and severity of significant medical comorbidities were scored using a web-based calculator (http://www.pmidcalc. org/?sid=7722560\&newtest $=$ Y\#\#ath) of the Charlson comorbidity index [17]. Information about recurrent urinary tract and pulmonary infections was also registered.

\section{Clinical features of COVID-19}

Collected clinical information about COVID-19 included common symptoms and signs, such as fever (temperature $\geq$ $37.5^{\circ} \mathrm{C}$ ), dry cough, dyspnea, diarrhea, anosmia, and fatigue. Pneumonia was classified as ground glass pattern or unilobar/multilobar, unilateral/bilateral, according to the appearance and distribution of lung consolidation(s) at the chest computed tomography scan. Data about the modified early warning score (MEWS) was also recorded. The MEWS is a scoring system assessing the deterioration risk in hospitalized patients, according to abnormal changes in physiologic parameters such as vital signs or mental status [18]; a MEWS score $\geq 3$ indicated severe COVID-19.

\section{Statistical analysis}

Data were presented as median (25th-75th percentile) for continuous variables and as percentage for categorical variables. After assessing the non-normal distribution of data with Shapiro-Wilk test, the differences between studied groups were evaluated with Wilcoxon rank sum test or Fisher exact test, as appropriate. All analyses were performed with $\mathrm{R}$ statistical software (version 3.6.3, The R Foundation for Statistical Computing, Vienna, Austria).

\section{Results}

On April 30, 2020, all the 25 SCI patients admitted to the inpatient rehabilitation center had been tested for SARSCoV-2 by the nasopharyngeal swab and 15 of them had a positive result. Also, 17 out of the 69 healthcare workers of the same center tested positive for SARS-CoV-2. The main clinical characteristics of SARS-Cov-2-positive SCI patients are presented in Table 1. Overall, 5 out of 15 (33.3\%) SARS-CoV-2-positive patients with SCI were asymptomatic: this group did not exhibit significant differences in clinical characteristics with respect to symptomatic SCI patients (Table 1). 
Table 1 Demographic, clinical, and SCI-related characteristics of the 15 SARS-CoV-2-positive patients with SCI categorized according to the presence and absence of COVID-19 symptoms.

\begin{tabular}{|c|c|c|c|}
\hline \multirow[t]{2}{*}{ Characteristics } & \multicolumn{2}{|c|}{ COVID-19 symptoms } & \multirow[t]{2}{*}{$p$ value } \\
\hline & Yes $(n=10)$ & No $(n=5)$ & \\
\hline \multicolumn{4}{|l|}{ Physiological and general clinical variables } \\
\hline Age (years) & $60.5(54.2-70.0)$ & $57.0(49.0-67.0)$ & 0.39 \\
\hline Males—n $(\%)$ & $7(70 \%)$ & $3(60 \%)$ & 1 \\
\hline Body mass index $\left(\mathrm{kg} / \mathrm{m}^{2}\right)$ & $26.6(25.4-27.9)$ & $24.5(23.8-29.1)$ & 0.75 \\
\hline Current smokers- $n(\%)$ & $2(20 \%)$ & $1(20 \%)$ & 1 \\
\hline Charlson comorbidity index & $1.0(0.0-2.0)$ & $0.0(0.0-2.0)$ & 0.84 \\
\hline Patients with recurrent UTIs- $n(\%)$ & $4(40 \%)$ & $1(20 \%)$ & 0.61 \\
\hline Chronic lung diseases $-n(\%)$ & $0(0 \%)$ & $0(0 \%)$ & 1 \\
\hline Metabolic syndrome— $n(\%)$ & $6(60 \%)$ & $2(40 \%)$ & 0.61 \\
\hline Hypertension diagnosis and/or treatment- $n(\%)$ & $5(50 \%)$ & $1(20 \%)$ & 0.58 \\
\hline Dyslipidemia diagnosis and/or treatment- $n(\%)$ & $4(40 \%)$ & $1(20 \%)$ & 0.61 \\
\hline T2DM diagnosis and/or treatment- $n(\%)$ & $2(20 \%)$ & $1(20 \%)$ & 1 \\
\hline Cancer diagnosis- $n(\%)$ & $1(10 \%)$ & $1(20 \%)$ & 1 \\
\hline Chronic heart failure $-n(\%)$ & $1(10 \%)$ & $0(0 \%)$ & 1 \\
\hline \multicolumn{4}{|l|}{ Injury-related variables } \\
\hline Duration of injury (months) & $3.0(2.0-33.0)$ & $96.0(12.0-118.0)$ & 0.32 \\
\hline Complete motor lesion (AIS A $+\mathrm{B})-n(\%)$ & $5(50 \%)$ & $1(20 \%)$ & 0.58 \\
\hline Incomplete motor lesion (AIS C + D)— $-n(\%)$ & $5(50 \%)$ & $4(80 \%)$ & 0.57 \\
\hline \multicolumn{4}{|l|}{ SCI level— $n(\%)$} \\
\hline Cervical & $5(50 \%)$ & $3(60 \%)$ & 1 \\
\hline Thoracic & $4(40 \%)$ & $1(20 \%)$ & 0.60 \\
\hline Lumbosacral & $1(10 \%)$ & $1(20 \%)$ & 1 \\
\hline SCIM score & $31.0(20.3-52.0)$ & $71.0(68.0-72.0)$ & 0.07 \\
\hline Tracheostomy— $n(\%)$ & $0(0 \%)$ & $0(0 \%)$ & 1 \\
\hline Mechanical aspiration respiratory secretion $-n(\%)$ & $0(0 \%)$ & $0(0 \%)$ & 1 \\
\hline
\end{tabular}

Values were medians (25th-75th percentile) for continuous variables and number (percentage) for categorical variables.

AIS ASIA (American Spinal Injury Association) Impairment Scale, COVID-19 coronavirus disease 2019, SARS-CoV-2 severe acute respiratory syndrome coronavirus 2, SCI spinal cord injury, SCIM spinal cord independence measure, T2DM type 2 diabetes mellitus, UTIs urinary tract infections.
As shown in Table 2, when individuals with SCI and able-bodied healthcare workers were compared, the SCI group exhibited a significantly more advanced age and a higher prevalence of comorbidities, as assessed by the Charlson comorbidity index. However, no significant differences in COVID-19 clinical features and treatment strategies between the two groups were observed. In particular, tocilizumab (dosage $8 \mathrm{mg} / \mathrm{kg}$ ) was used only in two male participants within the group of healthcare workers. Furthermore, the MEWs did not reveal severe COVID-19 in any of the subjects. All hospitalized subjects were treated in nonintensive care units and no deaths occurred in either group (Table 2).

\section{Discussion}

With the spread of the COVID-19 pandemic, serious concerns are arising for individuals with SCI [4, 5, 19]. Although concerns could be justified by the peculiarities of this population, some reassuring findings have been produced in our series of individuals with SCI who did not exhibit significant differences in clinical features and evolution of COVID-19 when compared with able-bodied individuals.

A matter of concern in people with SCI is that consequences of neurological damage could mask the clinical expression of features commonly suggestive of COVID-19 in the general population. Of note, in our series, the proportion of asymptomatic cases was higher, albeit not significantly different, in SCI patients (5 out of 15) than in able-bodied control group ( 2 out of 17); this could reflect a milder clinical course of COVID-19 in SCI or otherwise the impairment of cough reflex and thermoregulation mechanisms, which is commonly seen following SCI, especially in the presence of a rostral cervical neurological lesion. Indeed, most of the patients from our series had incomplete lesions, making respiratory muscles and thermoregulation less affected. Interestingly, no significant differences in demographic, clinical, and SCI-related characteristics between symptomatic and asymptomatic patients were found and dry cough was the second most common symptom of COVID-19 without significant differences between people with SCI and able-bodied controls. This 
Table 2 Main clinical characteristics of COVID-19 in individuals with SCI and able-bodied controls.

\begin{tabular}{|c|c|c|c|}
\hline & $\begin{array}{l}\text { SCI group } \\
(n=15)\end{array}$ & $\begin{array}{l}\text { Healthcare } \\
\text { workers } \\
(n=17)\end{array}$ & $p$ value \\
\hline Age (years) & $58.0(53.5-59.0)$ & $45.0(41.8-48.2)$ & 0.03 \\
\hline Males-n $(\%)$ & $10(66.7 \%)$ & $5(29.4 \%)$ & 0.08 \\
\hline $\begin{array}{l}\text { Charlson comorbidity index } \geq \\
1-n(\%)\end{array}$ & $8(53.4 \%)$ & $0(0.0 \%)$ & 0.0001 \\
\hline \multicolumn{4}{|l|}{ COVID-19 clinical features $-n(\%)$} \\
\hline Symptomatic & $10(66.7 \%)$ & $15(88.2 \%)$ & 0.21 \\
\hline Fever & $10(66.7 \%)$ & $14(82.4 \%)$ & 0.24 \\
\hline Dry cough & $9(60.0 \%)$ & $11(64.7 \%)$ & 1 \\
\hline Dyspnea & $3(20.0 \%)$ & $7(41.2 \%)$ & 0.14 \\
\hline Diarrhea & $2(13.4 \%)$ & $7(41.2 \%)$ & 0.24 \\
\hline Anosmia & $1(6.7 \%)$ & $4(23.5 \%)$ & 0.36 \\
\hline Fatigue & $2(13.4 \%)$ & $3(17.6 \%)$ & 1 \\
\hline MEWS $\geq 3-n(\%)$ & $0(0.0 \%)$ & $0(0.0 \%)$ & 1 \\
\hline \multicolumn{4}{|c|}{ COVID-19 radiological findings $-n(\%)$} \\
\hline Unilobar/Multilobar pneumonia & $1(6.7 \%)$ & $2(11.8 \%)$ & 0.73 \\
\hline Ground glass pattern & $8(53.4 \%)$ & $7(41.2 \%)$ & 0.72 \\
\hline \multicolumn{4}{|l|}{ COVID-19 treatment- $n(\%)$} \\
\hline Low-flow oxygen $(\leq 4 \mathrm{~L} / \mathrm{min})$ & $9(60.0 \%)$ & $4(23.5 \%)$ & 0.07 \\
\hline High-flow oxygen (>4 L/min) & $0(0.0 \%)$ & $1(5.9 \%)$ & 0.98 \\
\hline Lopinavir/Ritonavir (200/50 mg) & $8(53.4 \%)$ & $7(41.2 \%)$ & 0.72 \\
\hline Tocilizumab $(8 \mathrm{mg} / \mathrm{kg})$ & $0(0.0 \%)$ & $2(11.8 \%)$ & 0.48 \\
\hline $\begin{array}{l}\text { Hydroxychloroquine/ } \\
\text { Azithromycin/Heparin LMW }\end{array}$ & $10(66.7 \%)$ & $7(41.2 \%)$ & 0.17 \\
\hline \multicolumn{4}{|l|}{ COVID-19 evolution- $n(\%)$} \\
\hline Nonintensive care unit & $8(53.4 \%)$ & $7(41.2 \%)$ & 0.72 \\
\hline Intensive care unit & $0(0.0 \%)$ & $0(0.0 \%)$ & 1 \\
\hline Deaths & $0(0.0 \%)$ & $0(0.0 \%)$ & 1 \\
\hline
\end{tabular}

Values were medians (25th-75th percentile) for continuous variables and numbers (percentage) for categorical variables.

COVID-19 coronavirus disease 2019, LMW low molecular weight, MEWS modified early warning score.

finding is consistent with the results from a small series of seven SARS-CoV-2-positive patients with SCI, where cough was reported in up to $50 \%$ of cases [12]. As a result of sympathetic denervation that results in deregulation of body temperature, people with SCI also can exhibit low body temperature at rest as well as a poor febrile response to inflammatory and infectious diseases [19]. In this scenario, COVID-19 could go unnoticed because of the lack of fever. On the contrary, in the present study, all symptomatic SARS-Cov-2-positive individuals with SCI had fever. In keeping with this finding, in the only other case series of people with SCI so far reported, six out of seven patients with COVID-19 displayed fever [12].

Concern about a possible delay in COVID-19 diagnosis due to SCI-related clinical confounding factors lies in the overall frailty peculiar of individuals with SCI. People with SCI are at increased risk of a wide spectrum of comorbidities, which, in turn, represent well-documented negative prognostic factors for COVID-19, especially type 2 diabetes mellitus, hypertension, and cardiovascular disease [2].
People with SCI experience a dramatic change in their body composition along with an impairment of metabolic homeostasis. The denervation of the sublesional somatic levels leads to significant muscle hypotrophy, resulting in the reduction of energy expenditure and increase in fat mass [20]: this could explain the increased odds for developing cardiovascular diseases, type 2 diabetes mellitus, and hypertension [7-10]. Accordingly, in our series, a substantial percentage of SARS-CoV-2-positive individuals with SCI displayed metabolic syndrome (53.4\%), hypertension (40\%), dyslipidemia (33.6\%), and type 2 diabetes mellitus (20\%). Nevertheless, quite unexpectedly, COVID19 did not show a worse course in SCI group with respect to able-bodied controls.

Following SCI, a high incidence of recurrent respiratory tract infections is also usually reported, reflecting the deep changes in respiratory physiology, especially in individuals with tetraplegia $[4,5,19]$. In addition, immunological alterations in chronic and subacute SCI could contribute to the high susceptibility to severe pulmonary infections $[4,5,19]$. Among the 25 patients with SCI admitted to our inpatient rehabilitation center, only two males had a tracheotomy and needed mechanical respiratory support at the time of the national lockdown declaration, but, fortunately, they tested negative for SARS-CoV-2 in two consecutive swabs, and were immediately isolated. However, although in our series of SARS-CoV-2positive SCI patients no cases of preexistent history of chronic lung diseases or infections were reported, up to $60 \%$ of them required oxygen therapy, which, instead, was used only in $29.4 \%$ of the healthcare staff controls. Even if the difference did not reach statistical significance, the need for the implementation of respiratory medical management during infection could reflect a higher vulnerability of SCI population to the detrimental effects of SARS-CoV-2 on the lung.

Overall, in our series, SCI patients had a favorable prognosis and did not differ from controls. A possible explanation could lie in the early detection and management of COVID-19, as they represent positive prognostic factors even in special frail populations. Moreover, changes in endocrine homeostasis may also have influenced the clinical course of the infection in our SCI population. Men with SCI exhibit a very high prevalence of androgen deficiency [21-23] and, intriguingly, testosterone is a key regulator of the transmembrane serine protease 2 (TMPRSS2) [24], an androgen-dependent protease required for SARS-CoV-2 activation and spread in the respiratory epithelial cells [25]. In this light, it can be speculated that low testosterone levels could result in poor TMPRSS2 activity, thus representing a "protective factor" against a worse COVID-19 clinical evolution. Unfortunately, the small sample size of the study population hindered adjustments of the analysis for testosterone levels in male subgroups. However, the actual role of testosterone remains to be clarified as, in a recent study, low 
testosterone levels predicted both poor prognosis and mortality in men with COVID-19 admitted to respiratory intensive care unit [26].

This study has some limitations. First, the small sample size of enrolled subjects in both groups. However, in the context of the epidemiological dimension of SCI [27], in our knowledge, this is the largest series of SCI individuals with COVID-19 reported so far in the literature. Furthermore, as a relevant strength, this is the first study also including an able-bodied control group, which was recruited within the same center. Second, although the proportion of positive nasopharyngeal swabs was higher in SCI group (15 out of 25) than in healthcare workers (17 out of 69), the cross-sectional design of the study did not allow us to draw conclusions regarding possible differences in vulnerability to acquire the SARS-CoV2 infection; indeed, all individuals were enrolled after diagnosis and exposure conditions of SCI patients and healthcare workers were not comparable. Third, laboratory data with a documented value in predicting severity and evolution of COVID-19 were lacking in our series; this is understandable in the context of a widespread outbreak, also involving most of the healthcare staff. Finally, a comparison bias could arise from the younger age and predominance of female gender in the healthcare workers group. Nevertheless, these differences would reinforce the evidence that SCI is not associated to a worse COVID-19 clinical evolution, as both older age and male gender seem to be negative prognostic factors [2].

In conclusion, despite the high prevalence of comorbidities and poor overall health status in people with SCI, the present study suggested that COVID-19 does not exhibit atypical clinical features or a worse evolution in this frail population when compared with able-bodied individuals. These findings can highlight the value of the early diagnosis, allowing a close medical follow-up and a prompt treatment. Larger studies are warranted to confirm our observation and define prognostic predictors in SARS-CoV-2 infected people with SCI.

\section{Compliance with ethical standards}

Conflict of interest The authors declare that they have no conflict of interest.

Publisher's note Springer Nature remains neutral with regard to jurisdictional claims in published maps and institutional affiliations.

\section{References}

1. Wang C, Horby PW, Hayden FG, Gao GF. A novel coronavirus outbreak of global health concern. Lancet. 2020;395:470-3.

2. Rodriguez-Morales AJ, Cardona-Ospina JA, Gutiérrez-Ocampo E, Villamizar-Peña R, Holguin-Rivera Y, Escalera-Antezana JP, et al. Clinical, laboratory and imaging features of COVID-19: a systematic review and meta-analysis. Travel Med Infect Dis. 2020;34:101623.
3. European CDC. Situation update worldwide. European CDC; 2020. OurWorldInData.org/coronavirus.

4. Korupolu R, Stampas A, Gibbons C, Hernandez Jimenez I, Skelton F, Verduzco-Gutierrez M. COVID-19: screening and triage challenges in people with disability due to spinal cord injury. Spinal Cord Ser Cases. 2020;6:35.

5. López-Dolado E, Gil-Agudo A. Lessons learned from the coronavirus disease 2019 (Covid-19) outbreak in a monographic center for spinal cord injury. Spinal Cord. 2020;58:517-9.

6. Garshick E, Kelley A, Cohen SA, Garrison A, Tun CG, Gagnon $\mathrm{D}$, et al. A prospective assessment of mortality in chronic spinal cord injury. Spinal Cord. 2005;43:408-16.

7. Cragg JJ, Noonan VK, Krassioukov A, Borisoff J. Cardiovascular disease and spinal cord injury: results from a national population health survey. Neurology. 2013;81:723-8.

8. Gater DR, Farkas GJ, Berg AS, Castillo C. Prevalence of metabolic syndrome in veterans with spinal cord injury. J Spinal Cord Med. 2019;42:86-93.

9. Cragg JJ, Noonan VK, Dvorak M, Krassioukov A, Mancini GB, Borisoff JF. Spinal cord injury and type 2 diabetes: results from a population health survey. Neurology. 2013;81:1864-8.

10. Bauman WA. The potential metabolic consequences of cerebral palsy: inferences from the general population and persons with spinal cord injury. Dev Med Child Neurol. 2009;51:64-78.

11. Righi G, Del Popolo G. COVID-19 tsunami: the first case of a spinal cord injury patient in Italy. Spinal Cord Ser Cases. 2020;6:22.

12. Rodríguez-Cola M, Jiménez-Velasco I, Gutiérrez-Henares F, López-Dolado E, Gambarrutta-Malfatti C, Vargas-Baquero E, et al. Clinical features of coronavirus disease 2019 (COVID-19) in a cohort of patients with disability due to spinal cord injury. Spinal Cord Ser Cases. 2020;6:39.

13. World Health Organization. WHO Director-General's opening remarks at the media briefing on COVID-19-11 March 2020. World Health Organization; 2020. https://www.who.int/dg/ speeches/detail/who-director-general-s-opening-remarks-atthemedia-briefing-on-covid-19--11-march-2020.

14. von Elm E, Altman DG, Egger M, Pocock StuartJ, Gøtzsche PeterC, Vandenbroucke JanP, STROBE Initiative. The strengthening the reporting of observational studies in epidemiology (STROBE) statement: guidelines for reporting observational studies. Lancet. 2007;370:1453-7.

15. Maynard FM, Bracken MB, Creasey G, Ditunno JF, Donovan WH, Ducker TB, et al. International standards for neurological and functional classification of spinal cord injury. Spinal Cord. 1997;35: 266-74.

16. Itzkovich M, Gelernter I, Biering-Sorensen F, Weeks C, Laramee MT, Craven BC, et al. The Spinal Cord Independence Measure (SCIM) version III: reliability and validity in a multi-center international study. Disabil Rehabil. 2007;29:1926-33.

17. Charlson M, Szatrowski TP, Peterson J, Gold J. Validation of a combined comorbidity index. J Clin Epidemiol. 1994;47:1245-51.

18. Subbe CP, Kruger M, Rutherford P, Gemmel L. Validation of a modified Early Warning Score in medical admissions. QJM. 2001;94:521-6.

19. Dicks MA, Clements ND, Gibbons CR, Verduzco-Gutierrez M, Trbovich M. Atypical presentation of Covid-19 in persons with spinal cord injury. Spinal Cord Ser Cases. 2020;6:38.

20. Laughton GE, Buchholz AC, Martin Ginis KA, Goy RE. Lowering body mass index cutoffs better identifies obese persons with spinal cord injury. Spinal Cord. 2009;47:757-62.

21. Bauman WA, La Fountaine MF, Spungen AM. Age-related prevalence of low testosterone in men with spinal cord injury. J Spinal Cord Med. 2014;37:32-39.

22. Barbonetti A, Caterina Vassallo MR, Cotugno M, Felzani G, Francavilla S, Francavilla F. Low testosterone and non-alcoholic 
fatty liver disease: evidence for their independent association in men with chronic spinal cord injury. J Spinal Cord Med. 2016;39:443-9.

23. Barbonetti A, Vassallo MRC, Felzani G, Francavilla S, Francavilla F. Association between $25(\mathrm{OH})$-vitamin $\mathrm{D}$ and testosterone levels: evidence from men with chronic spinal cord injury. J Spinal Cord Med. 2016;39:246-52.

24. Mikkonen L, Pihlajamaa P, Sahu B, Zhang FP, Jänne OA. Androgen receptor and androgen-dependent gene expression in lung. Mol Cell Endocrinol. 2010;317:14-24.
25. Stopsack KH, Mucci LA, Antonarakis ES, Nelson PS, Kantoff PW. TMPRSS2 and COVID-19: serendipity or opportunity for intervention? Cancer Discov. 2020;10:779-82.

26. Rastrelli G, Di Stasi V, Inglese F, Beccaria M, Garuti M, Di Costanzo D, et al. Low testosterone levels predict clinical adverse outcomes in SARS-CoV-2 pneumonia patients. Andrology. 2020. https://doi.org/10.1111/andr.12821.

27. Celani MG, Spizzichino L, Ricci S, Zampolini M, Franceschini M. Spinal cord injury in Italy: a multicenter retrospective study. Arch Phys Med Rehabil. 2001;82:589-96. 